\title{
Genetic and epigenetic alterations are involved in the regulation of TPM1 in cholangiocarcinoma
}

\author{
WEI YANG, XIAOYUAN WANG, WEI ZHENG, KEDONG LI, HAOFENG LIU and YUEMING SUN \\ Department of Minimally Invasive Surgery, The First Affiliated Hospital \\ of Nanjing Medical University, Nanjing 210029, P.R. China
}

Received October 6, 2012; Accepted November 22, 2012

DOI: 10.3892/ijo.2012.1741

\begin{abstract}
Cholangiocarcinoma is a malignant tumor originating from biliary epithelial cells. The tumor suppressor gene tropomyosin 1 (TPM1) is downregulated in several human cancer types; however, its expression status in cholangiocarcinoma is still unknown. We elucidated TPM1 expression and its regulation mechanism in cholangiocarcinoma. Real-time (RT)-PCR, western blot analysis and immunohistochemistry were performed to examine TPM1 expression levels in cholangiocarcinoma cell lines and tumor tissues. Cell lines were treated with lentiviral vector containing the miR-21 knockdown and inhibitors of genetic and epigenetic mechanisms (manumycin A, LY294002, U0126, DAC and TSA), and the TPM1 expression change was observed by RT-PCR and western blot analyses. Cell proliferation, apoptosis and migration were evaluated by water-soluble tetrazolium salt (WST-1) assay, flow cytometry and wound healing experiments, respectively. TPM1 was downregulated in the intrahepatic cholangiocarcinoma cells (HuCCT1) and upregulated in the extrahepatic cholangiocarcinoma cells (QBC939) compared with normal intrahepatic biliary epithelial cells (HIBEC). TPM1 stained negative in the intrahepatic cholangiocarcinoma tissues, as revealed by immunohistochemistry, although there was no significant difference in staining of the intrahepatic cholangiocarcinoma tissues and adjacent non-cancer tissues. RAS and two important downstream signaling pathways (RAS/ $\mathrm{PI} 3 \mathrm{~K} / \mathrm{AKT}$ and RAS/MEK/ERK) were involved in TPM1 regulation and inhibition of the epigenetic mechanisms such as DNA methylation, histone deacetylation and miR-21 upregulation upregulated TPM1 expression. Inhibitors of genetic and epigenetic mechanisms (manumycin A, LY294002, U0126, DAC and TSA) inhibited cell proliferation and migration and induced apoptosis. These data indicated that TPM1 is down-
\end{abstract}

Correspondence to: Dr Yueming Sun, Department of Minimally Invasive Surgery, The First Affiliated Hospital of Nanjing Medical University, 300 Guangzhou Road, Nanjing 210029, P.R. China E-mail: jssym@vip.sina.com

Key words: tumor suppressor gene tropomyosin 1, RAS, DNA methylation, histone deacetylation, miR-21, cholangiocarcinoma regulated in HuCCT1 cells and that the Ras signaling pathway as well as DNA methylation, histone deacetylation and miR-21 upregulation play important roles in the suppression of TPM1 expression in HuCCT1 cells. Thus, compounds that inhibit genetic and epigenetic mechanisms may be promising agents in treating cholangiocarcinoma.

\section{Introduction}

Cholangiocarcinoma is a significantly devastating tumor, is difficult to diagnose, and has a high mortality rate. The incidence of cholangiocarcinoma is increasing worldwide (1). In recent years, the 1- and 2-year survival rates were reported to be 24.5 and $12.8 \%$, respectively. As a result, this tumor continues to be associated with a poor prognosis. Cholangiocarcinoma is classified into three broad categories, namely intrahepatic, perihilar and distal tumors. Resection is considered as the best treatment, radiation or chemotherapy has no influence on survival and many patients do not get the opportunity to undergo surgery (2). Curative surgeries such as liver resection and liver transplantation for some hilar cholangiocarcinomas can achieve long-term survival. However, there are no specific tumor markers for early diagnosis; a combination of serum tumor markers that can identify most occult tumors is used to select appropriate cases for liver transplantation (3). Therefore, it is of great value to identify specific tumor markers and novel chemical agents for the diagnosis and treatment of cholangiocarcinoma and to improve patient survival.

Tropomyosins (TMs) are found as a family of actin filament-binding proteins in the skeletal muscle. The TM family is divided into two major groups: the high molecular weight (HMW) and the low molecular weight (LMW) tropomyosins. Tropomyosin 1 (TPM1) is a gene that encodes isoforms of the HMW TMs (4). The non-muscle cells express multiple isoforms of TMs, which are involved in cell transformation and differentiation and in the regulation of actin filament stability (5). HMW TM isoforms are downregulated in some tumors, such as breast, urinary bladder and neuroblastoma tumors (6-9). HMW TMs can regulate the proliferation, motility, invasion, and metastasis of tumor cells (10). TPM1 encodes most important TMs in breast cancer, where it functions as a suppressor of cell transformation (11). In the present study, we aimed to determine the expression levels of TPM1 and its regulation mechanism in cholangiocarcinoma. 


\section{Materials and methods}

Patients and cell lines. Intrahepatic cholangiocarcinoma tissue and adjacent non-cancer tissue were surgically obtained between January 2010 and May 2012 from 30 patients at The First Affiliated Hospital of Nanjing Medical University. Written informed consent was obtained from all the patients before sample collection. The intrahepatic cholangiocarcinoma cells (HuCCT1), extrahepatic cholangiocarcinoma cells (QBC939) and the normal intrahepatic biliary epithelial cells (HIBEC) obtained from American Type Culture Collection (ATCC; Rockville, MD) were grown in Dulbecco's medium essential medium (DMEM; Gibco, Grand Island, NY, USA) containing $10 \%$ fetal bovine serum (FBS), penicillin (100 U/ml), and streptomycin $(100 \mu \mathrm{g} / \mathrm{ml})$ under cell culture conditions $\left(5 \% \mathrm{CO}_{2}, 95 \%\right.$ relative humidity, and $\left.37^{\circ} \mathrm{C}\right)$.

Quantitative real-time ( $R T)$-PCR for TPM1. Total RNA was purified from cultured cells using the RNAiso ${ }^{\mathrm{TM}}$ Plus kit (Takara, Tokyo, Japan). cDNA was synthesized using the PrimeScript RT Master Mix (Takara) in a $10-\mu l$ reaction mixture according to the manufacturer's protocol. cDNA $(2 \mu \mathrm{l})$ was used as a template in a $20-\mu l$ reaction using the SYBR Premix Ex Taq real-time PCR kit (Takara). Glyceraldehyde-3phosphate dehydrogenase (GAPDH) was used as the reference gene. The following primers were used for the detection of TPM1 expression: 5'-ACGAACAACTTGAAGTCACTAA-3' (sense) and 5'-TTTAGTTACTGACCTCTCCGCA-3' (antisense). The primers for GAPDH were: 5'-ACGGATTTGGTC GTATTGGGC-3' (sense) and 5'-TTGACGGTGCCATGG AATTTG-3' (antisense). PCR was performed using the following cycles: $95^{\circ} \mathrm{C}$ for $30 \mathrm{sec}, 40$ cycles of $95^{\circ} \mathrm{C}$ for $5 \mathrm{sec}$, $60^{\circ} \mathrm{C}$ for $31 \mathrm{sec}$ and the dissociation stage: $95^{\circ} \mathrm{C}$ for $15 \mathrm{sec}$, $60^{\circ} \mathrm{C}$ for $1 \mathrm{~min}$ and $95^{\circ} \mathrm{C}$ for $15 \mathrm{sec}$.

Western blot analysis. Cells were lysed, and equal amounts of protein were electrophoresed on a $12 \%$ SDS-polyacrylamide gel and subsequently transferred to polyvinylidene fluoride (PVDF) membranes. The membranes were blocked in 5\% skim milk in phosphate-buffered saline (PBS) containing $0.1 \%$ Tween-20 (PBST) for $1 \mathrm{~h}$ at room temperature. The membranes were incubated with the following primary antibodies at $4^{\circ} \mathrm{C}$ overnight: TPM1 (1:400; ab55915, Abcam, Hong Kong, China), $\beta$-actin (1:800; Beijing Biosynthesis Biotechnology, Beijing, China), and $\alpha$-tubulin (1:500; Beijing Biosynthesis Biotechnology). The membranes were then washed three times with PBST, incubated with horseradish peroxidase (HRP)-conjugated secondary antibody $(1: 2,000$; Beijing Biosynthesis Biotechnology) for $2 \mathrm{~h}$ at room temperature. After three PBST washes, the membranes were developed using ECL Plus (Millipore, MA, USA) and exposed to X-ray film for visualization of protein bands. $\beta$-actin and $\alpha$-tubulin were used as internal loading controls.

Immunohistochemistry. Immunohistochemistry was carried out on 30 cholangiocarcinoma tissues and 30 adjacent non-cancer tissues by overnight incubation at $4^{\circ} \mathrm{C}$ using a polyclonal antibody to TPM1 (ab55915, Abcam). The sections were subsequently treated with goat anti-mouse secondary antibody, followed by further incubation with streptavidin-HRP complex. Diaminobenzidine (Dako, Glostrup, Denmark) was used as a chromogen, and sections were lightly counterstained with hematoxylin. Immunostaining intensity $(0$, no staining; 1 , weak staining; 2 , moderate staining; 3 , strong staining) as well as the percentage of cells stained ( 0 , no cells; $1,<10 \%$ of cells; $2,11-50 \%$ of cells; $3,51-80 \%$ of cells; $4,>80 \%$ of cells) were evaluated, and the respective scores were multiplied, resulting in intensity values ranging from 0 to 12 . The tissues showing immunostaining intensity values in the rage (0-6) were grouped as negative and those showing immunostaining intensity values in the rage (7-12) were grouped as positive.

Taqman $^{\mathrm{TM}}$ quantitative RT-PCR for miR-21. Total RNA was extracted from cultured cells using the RNAiso Plus (Takara) and mature miR-21 was detected using the miRCURY LNA ${ }^{\mathrm{TM}}$ Universal RT microRNA PCR kit (Roche, Germany). The RT-PCR results were analyzed and expressed as relative $\mathrm{Ct}$ (threshold cycle) values, which were then converted to foldchanges. Each sample was measured in triplicate. The small nuclear RNA U6 (U6 snRNA) was used as an endogenous control.

Plasmid construction, lentivirus packaging, and infection. To construct a lentiviral vector (LV) containing the miR-21 knockdown (LV-anti-miR21), as well as the negative control (LV-GFP), a complementary sequence that targeted miR-21 was designed and two suitable PCR primers that targeted the miR-21 sequence were annealed in order to obtain the fragment insert. The PCR primers were as follows: forward 5'-CCGGT CAACATCAGTCTGATAAGCTATTTTTG-3' and reverse: 5'-AATTCAAAAATAGCTTATCAGACTGATGTTGA-3'. The fragment was cloned into the GV159 vector, after which the GV159 vector $(20 \mu \mathrm{g})$, the pHelper 1.0 vector $(15 \mu \mathrm{g})$, and the pHelper 2.0 vector (and $10 \mu \mathrm{g}$; Genechem Co., Shanghai, China) were cotransfected into 293T cells (purchased from ATCC) using $100 \mu 1$ Lipofectamine $^{\mathrm{TM}} 2000$ (Invitrogen, Carlsbad, CA, USA). The supernatants were collected $48 \mathrm{~h}$ later, were filtered (pore size, $0.45-\mu \mathrm{m}$ ) and stored at $-80^{\circ} \mathrm{C}$. HuCCT1 cells were infected with either LV-anti-miR21 or LV-green fluorescent protein (GFP) in the presence of $5 \mu \mathrm{g} / \mathrm{ml}$ polybrene (Sigma, St. Louis, MO, USA). The medium was refreshed after $6 \mathrm{~h}$, and the efficiency of infection was measured $72 \mathrm{~h}$ later under a fluorescent microscope based on GFP expression.

Cell proliferation assay. Cells were treated with manumycin A (Cayman Chemicals, MI, USA; 1, 5, 10, 25, 50 and $75 \mu \mathrm{mol} /$ ) for 24 and $48 \mathrm{~h}, \mathrm{LY} 294002$ (Sigma; 5, 10, 20 and $50 \mu \mathrm{mol}$ ) for 48 and $96 \mathrm{~h}, \mathrm{U} 0126$ (Sigma; 10, 20, 50 and $75 \mu \mathrm{mol}$ ) for 48 and 96 h, 5-aza-2-deoxycytidine (DAC) (Sigma; 0.1, 0.5, 1, 5, 25 and $100 \mu \mathrm{mol}$ ) for 24 and $48 \mathrm{~h}$ (DAC was replaced with fresh drug every $24 \mathrm{~h}$ ), and trichostatin A (TSA) (Sigma; 0.1, 0.25, $0.5,1.0,1.5$ and $2.0 \mu \mathrm{mol})$ for 24 and $48 \mathrm{~h}$.

Cells $\left(4 \times 10^{3}\right)$ were seeded in 96-well plates in $100 \mu \mathrm{l}$ medium containing 10\% FBS. After $24 \mathrm{~h}$, when the cells were grown to 50-60\% confluency, manumycin A, U0126, LY294002, DAC or TSA were added. Cell proliferation was assessed at 24 and $48 \mathrm{~h}$ or 48 and $96 \mathrm{~h}$ by using the Cell Counting kit-8 (CCK-8; Beyotime, Jiangsu, China) according to the manufacturer's protocol. 

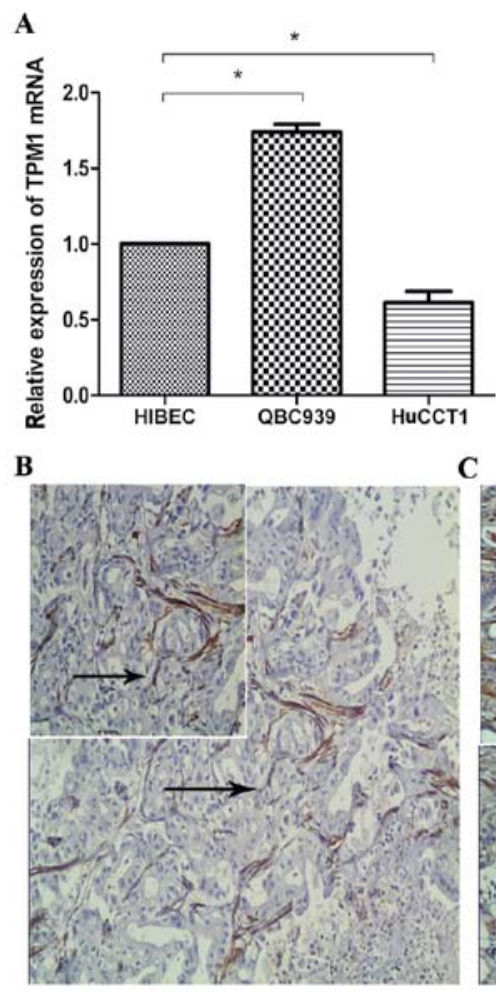

C

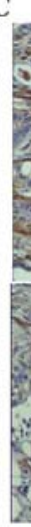

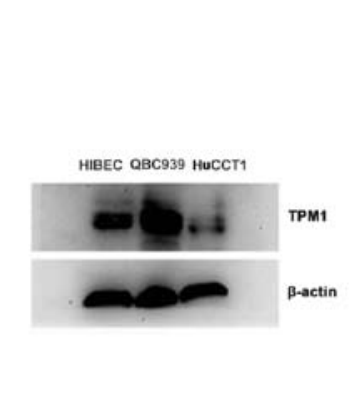

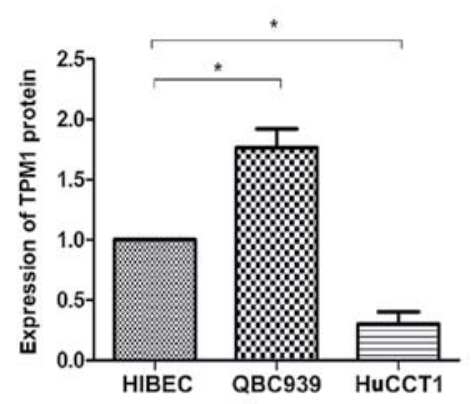

D

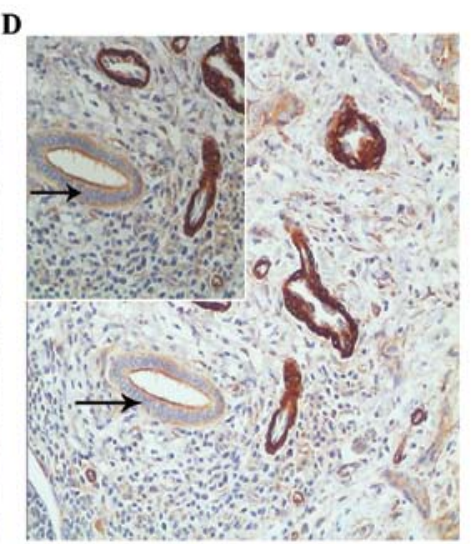

Figure 1. (A) TPM1 mRNA and protein expression in HuCCT1, QBC939 and HIBEC cells. (B and C) Staining of TPM1 in cholangiocarcinoma tissue. (D) Adjacent non-cancer tissue (200x; inset, 400x).

Apoptosis assays. Cells $\left(4 \times 10^{5}\right)$ were seeded in 6-well plates. After $24 \mathrm{~h}$, cells were treated with manumycin A $(10 \mu \mathrm{mol})$, $\mathrm{U} 0126(75 \mu \mathrm{mol})$ or TSA $(0.25 \mu \mathrm{mol})$ for $24 \mathrm{~h}$ and LY294002 (75 $\mu \mathrm{mol})$ and DAC $(25 \mu \mathrm{mol})$ for $48 \mathrm{~h}$. Apoptosis was detected using the Annexin V-FITC Apoptosis Detection kit (Keygen, Nanjing, China). Results were represented as the percentage of apoptotic cells in all the counted cells.

Wound healing experiments. Cells $\left(6 \times 10^{5}\right)$ were seeded in 6 -well plates. After $24 \mathrm{~h}$, when the cells were grown to $90-100 \%$ confluency, wounds were generated in cells by scraping with a plastic tip across the cell monolayer. The medium was replaced with serum-free medium, and the cells were treated with manumycin A $(5 \mu \mathrm{mol}), \mathrm{U} 0126(10 \mu \mathrm{mol}), \mathrm{LY} 294002(5 \mu \mathrm{mol}), \mathrm{DAC}$ $(25 \mu \mathrm{mol}), \mathrm{TSA}(0.1 \mu \mathrm{mol})$ for $24 \mathrm{~h}$. Phase-contrast images were recorded at the time of wounding $(0 \mathrm{~h})$ and at $24 \mathrm{~h}$ thereafter. Untreated cells served as controls.

Statistical analysis. Data were analyzed using the SPSS 13.0 software and are presented as mean \pm standard deviation (SD). Statistical comparisons between groups were performed using one-way analysis of variance (ANOVA) followed by a Student's t-test. $\mathrm{P}<0.05$ was considered statistically significant.

\section{Results}

TPM1 expression in cholangiocarcinoma cell lines and tissues. TPM1 expression was detected in HuCCT1 and QBC939 cell lines using western blot analysis and quantitative RT-PCR; HIBEC cells were used as a control. At the mRNA level, TPM1 was downregulated in HuCCT1 cells and upregulated in QBC939 cells compared with HIBEC cells (control). At the protein level, TPM1 was expressed at a much lower level in HuCCT1 cells compared with HIBEC cells, and was expressed at a higher level in QBC939 cells compared with HIBEC cells (Fig. 1A), indicating that the different expression of TPM1 in cholangiocarcinoma cells, is at the translational and transcriptional level. The immunohistochemistry results showed no staining (5/30) (Fig. 1B) or a weak staining (25/30) (Fig. 1C) for TPM1 in the intrahepatic cholangiocarcinoma tissues; however, all the adjacent non-cancer tissues (30/30) showed a weak staining for TPM1 (Fig. 1D). For statistical analysis, no staining and weak staining were grouped as negative and there was no significant difference between the staining results of intrahepatic cholangiocarcinoma tissues (0/30) and adjacent non-cancer tissues $(0 / 30)(\mathrm{P}>0.05)$.

Involvement of the Ras mutation in TPM1 downregulation. Previous studies have shown that the Ras signaling pathway is involved in TPM1 downregulation in urinary bladder carcinoma and breast cancer $(8,12)$. It is known that the K-ras gene is highly mutated in cholangiocarcinoma $(13,14)$. Furthermore, studies have also shown that the HuCCT1 cell line contains K-ras mutations that are located at codons 12 and 61, but not at codon 13 and that the mutated Ras family genes may contribute to the growth of cholangiocarcinoma $(15,16)$. The Ras family genes exert their functions by farnesylating themselves and manumycin offers promise as a specific inhibitor of the farnesylation process (17). It can exert an antiproliferative effect on human tumor cells that harbor a mutated K-ras gene (18). Based on the previous use of manumycin in abolishing the function of Ras in hepatocellular carcinoma (18) and pancreatic 

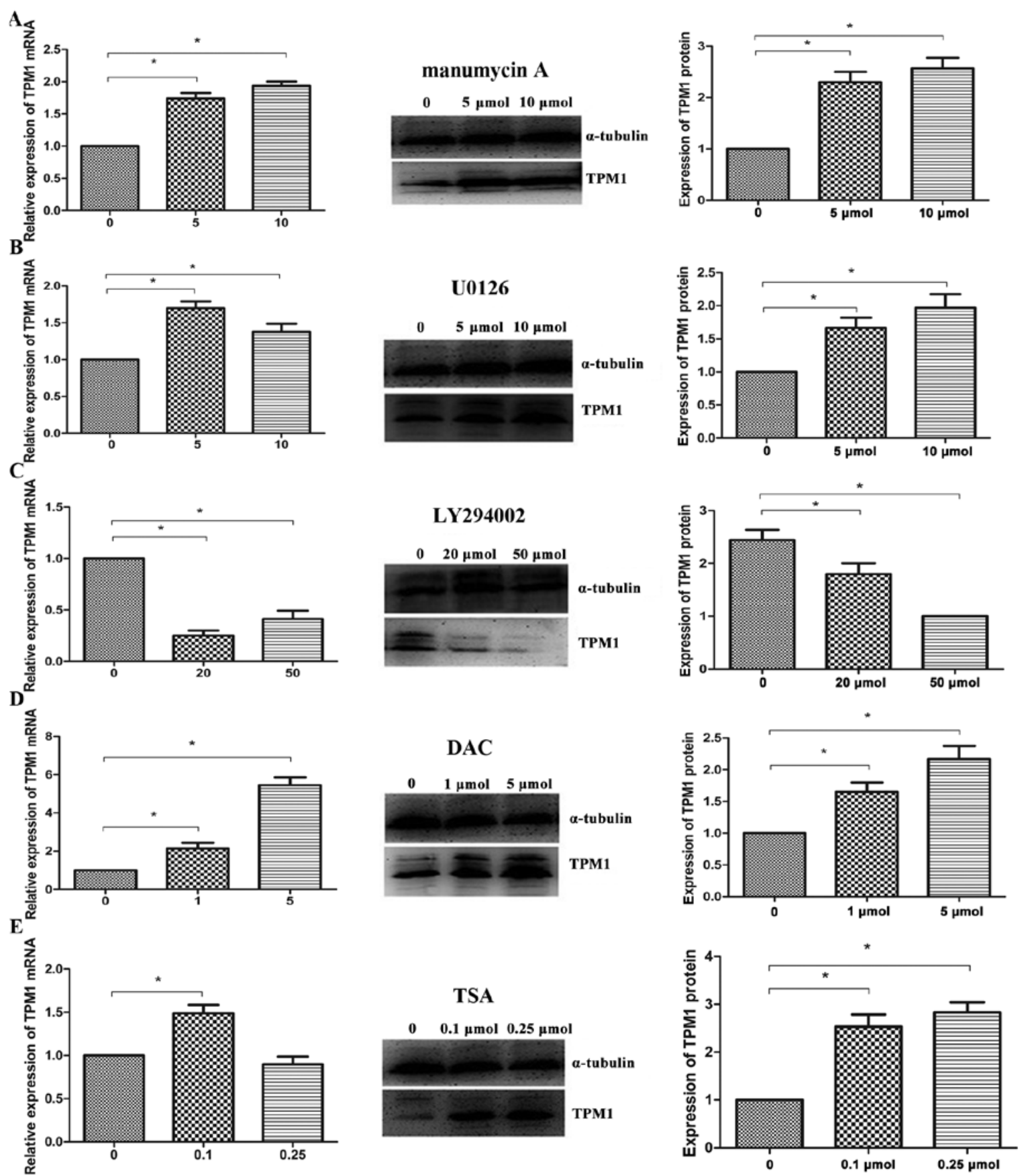

Figure 2. TPM1 mRNA expression and protein expression in HuCCT1 cells. (A) HuCCT1 cells were treated with 5 and $10 \mu$ mol/1 manumycin A for $24 \mathrm{~h}$. (B) HuCCT1 cells were treated with 5 and $10 \mu \mathrm{mol} / 1 \mathrm{U} 0126$ for $24 \mathrm{~h}$. (C) HuCCT1 cells were treated with 20 and $50 \mu \mathrm{mol} / 1 \mathrm{LY} 294002$ for $24 \mathrm{~h}$. (D) HuCCT1 cells were treated with 1 and $5 \mu \mathrm{mol} / 1 \mathrm{DAC}$ for $24 \mathrm{~h}$. (E) HuCCT1 cells were treated with 0.1 and $0.25 \mu \mathrm{mol} / 1 \mathrm{TSA}$ for $24 \mathrm{~h}$. "P<0.05.

cancer $(19,20)$, we treated HuCCT1 cells with manumycin A to determine whether the activated Ras can participate in the regulation of TPM1. Western blot analysis and RT-PCR of HuCCT1 cells, which were incubated with manumycin A ( 5 and $10 \mu \mathrm{mol} / \mathrm{l}$ ) for $24 \mathrm{~h}$, showed a significant restoration of TPM1 expression (Fig. 2A). This finding supports the role of activated Ras in the downregulation of TPM1.

Upregulation of TPMI by MEK inhibition. It has been shown that the RAS/MEK/ERK pathway participates in the downregulation of TPM1 $(8,12)$. In the present study, U0126, a specific MEK inhibitor, was used to investigate the role of this pathway in the downregulation of TPM1. HuCCT1 cells were incubated for $24 \mathrm{~h}$ with U0126 (5 and $10 \mu \mathrm{mol} / \mathrm{l})$. At the mRNA level, the TPM1 expression increased, as revealed by RT-PCR results. Similarly, western blot analysis suggested that U0126 can restore the TPM1 expression in HuCCT1 cells at the protein level when compared with that in untreated controls (Fig. 2B).

Involvement of the Ras/PI3K/AKT pathway in the expression of TPM1. Previous studies have reported that the PI3K/ AKT pathway does not affect the expression of TPM1 $(8,21)$. However, contradictory to this study, we found that the 


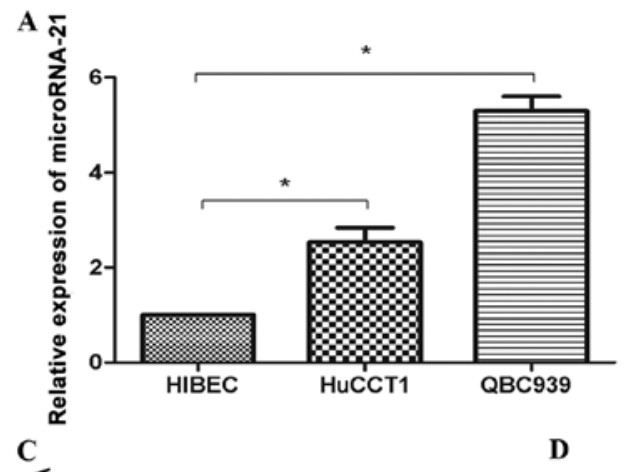

C

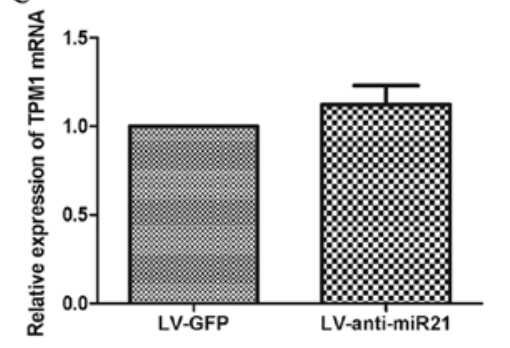

B

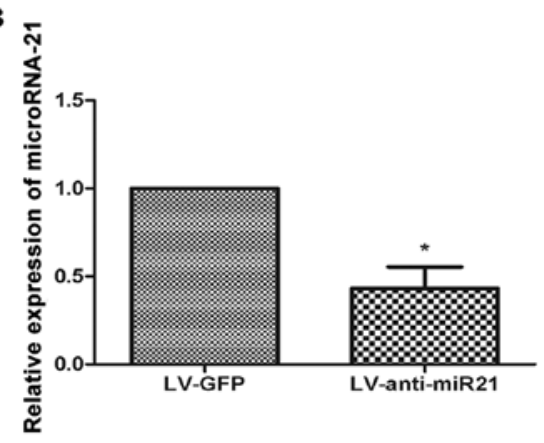

Figure 3. (A) miR-21 expression in cholangiocarcinoma cells (HuCCT1 and QBC939) compared with normal intrahepatic biliary epithelial cells (HIBEC). (B) Detection of miR-21 after HuCCT1 cells were infected with LV-anti-miR21 compared with LV-GFP-infected cells. (C) TPM1 mRNA expression after HuCCT1 cells were infected with LV-anti-miR21 compared with LV-GFP-infected cells. (D) TPM1 protein expression after HuCCT1 cells were infected with LV-anti-miR21 compared with LV-GFP-infected cells.

expression of TPM1 was decreased both at the mRNA level and at the protein level after a $24-\mathrm{h}$ treatment with the PI3K/ AKT-specific inhibitor LY294002 (20 and $50 \mu \mathrm{mol} / \mathrm{l})$, as shown by western blot analysis and RT-PCR (Fig. 2C).

DNA methylation and histone deacetylation are involved in the downregulation of TPM1. The present study showed that genetic mutations in the Ras signaling pathway play an important role in the regulation of TPM1 expression. The underlying epigenetic mechanisms have been found in breast cancer $(6,22)$ and melanoma (23). To elucidate the epigenetic mechanisms responsible for TPM1 regulation in cholangiocarcinoma, we treated HuCCT1 cells with the demethylating agent, DAC or with the histone deacetylase inhibitor, TSA and subsequently analyzed TPM1 expression using western blots and RT-PCR. Evaluation of TPM1 expression before and after treatment of HuCCT1 cells with DAC and TSA showed that TPM1 mRNA and protein expression (Fig. 2D) was significantly upregulated after a 24 -h treatment with DAC (1 and $5 \mu \mathrm{mol} / \mathrm{l})$ compared to untreated cells. The TPM1 mRNA expression was slightly upregulated in HuCCT1 cells after a 24-h treatment with $0.1 \mu \mathrm{mol} / 1 \mathrm{TSA}$, but not after a 24-h treatment with $0.25 \mu \mathrm{mol} / 1 \mathrm{TSA}$. However, contradictory to the mRNA levels, the TPM1 protein level in TSA-treated cells was significantly upregulated compared to untreated cells (Fig. 2E).

miR-21 is overexpressed in cholangiocarcinoma cell lines. TaqMan quantitative RT-PCR analysis demonstrated an overexpression of miR-21 in cholangiocarcinoma cell lines compared with non-malignant biliary epithelial cells (Fig. 3A). This result was in agreement with a previous study showing that miR-21 functions as an oncogene in cholangiocarcinoma (24). We also observed an inverse correlation between the levels of miR-21 and TPM1 in HuCCT1 cells, which suggests that miR-21 may also be involved in the loss of function of TPM1 in HuCCT1 cells.

miR-21 negatively regulates TPM1 protein levels in HuCCT1 cells. Previous studies reported that miR-21 promoted tumor growth through the downregulation of TPM1 in breast cancer $(25,26)$, tongue squamous cell carcinoma (27), prostate cancer (28) and glioma (29). In the present study, to investigate the relationship between miR-21 and TPM1 levels in HuCCT1 cells, a lentiviral vector containing the miR-21 knockdown (LV-anti-miR21) was constructed. In LV-anti-miR21-infected HuCCT1 cells, the miR-21 level was downregulated compared with that in LV-GFP-infected HuCCT1 cells (negative control) (Fig. 3B). RT-PCR and western blot analyses performed to investigate the effect of miR-21 on TPM1 expression showed that there was no difference in TPM1 expression in LV-antimiR21-infected or LV-GFP (negative control)-infected HuCCT1 cells at the RNA level. However, at the protein level, there was a significant increase in TPM1 expression in LV-antimiR21-infected HuCCT1 cells compared with that in LV-GFP (negative control)-infected HuCCT1 cells (Fig. 3C and D).

Inhibitor-induced inhibition of cell proliferation. In the present study, HuCCT1 cells were exposed to the inhibitors manumycin A, LY294002, U0126, DAC and TSA to investigate their effect on cell growth inhibition in cholangiocarcinoma. There are no previous reports on the use of manumycin in the treatment of cholangiocarcinoma. Results of the CCK- 8 assay showed that manumycin A inhibited HuCCT1 proliferation in a dose- and time-dependent manner (Fig. 4A). Because PI3K/ AKT and MEK/ERK are two important signaling pathways in cholangiocarcinoma $(30,31)$, the PI3K inhibitor LY294002 and the MEK inhibitor U0126 have been previously used to investigate their antiproliferative effect on cholangio- 
A

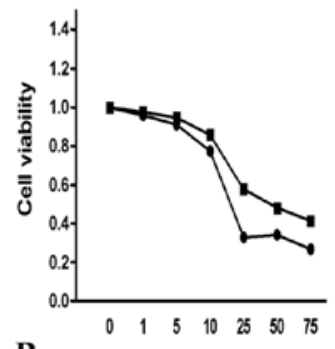

B

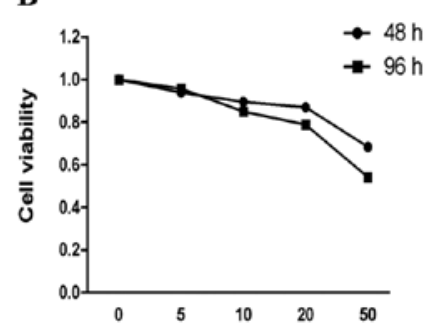

C

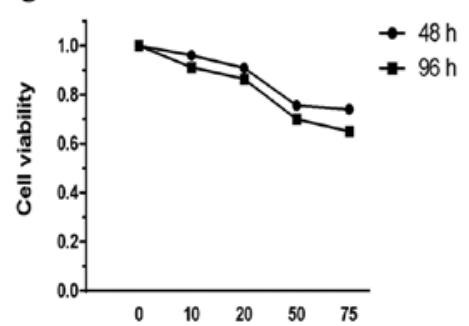

D

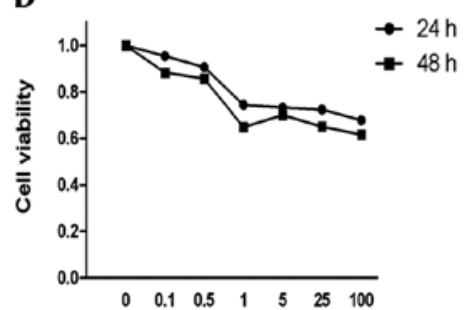

$\mathbf{E}$

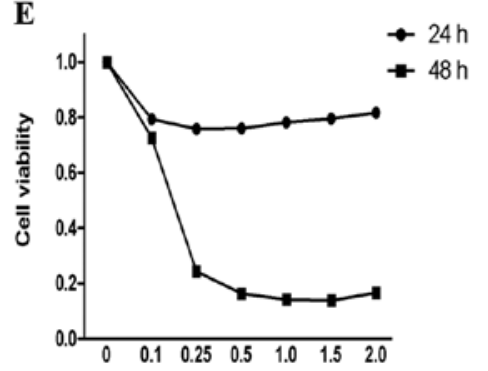

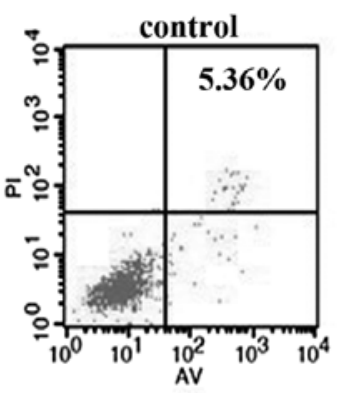
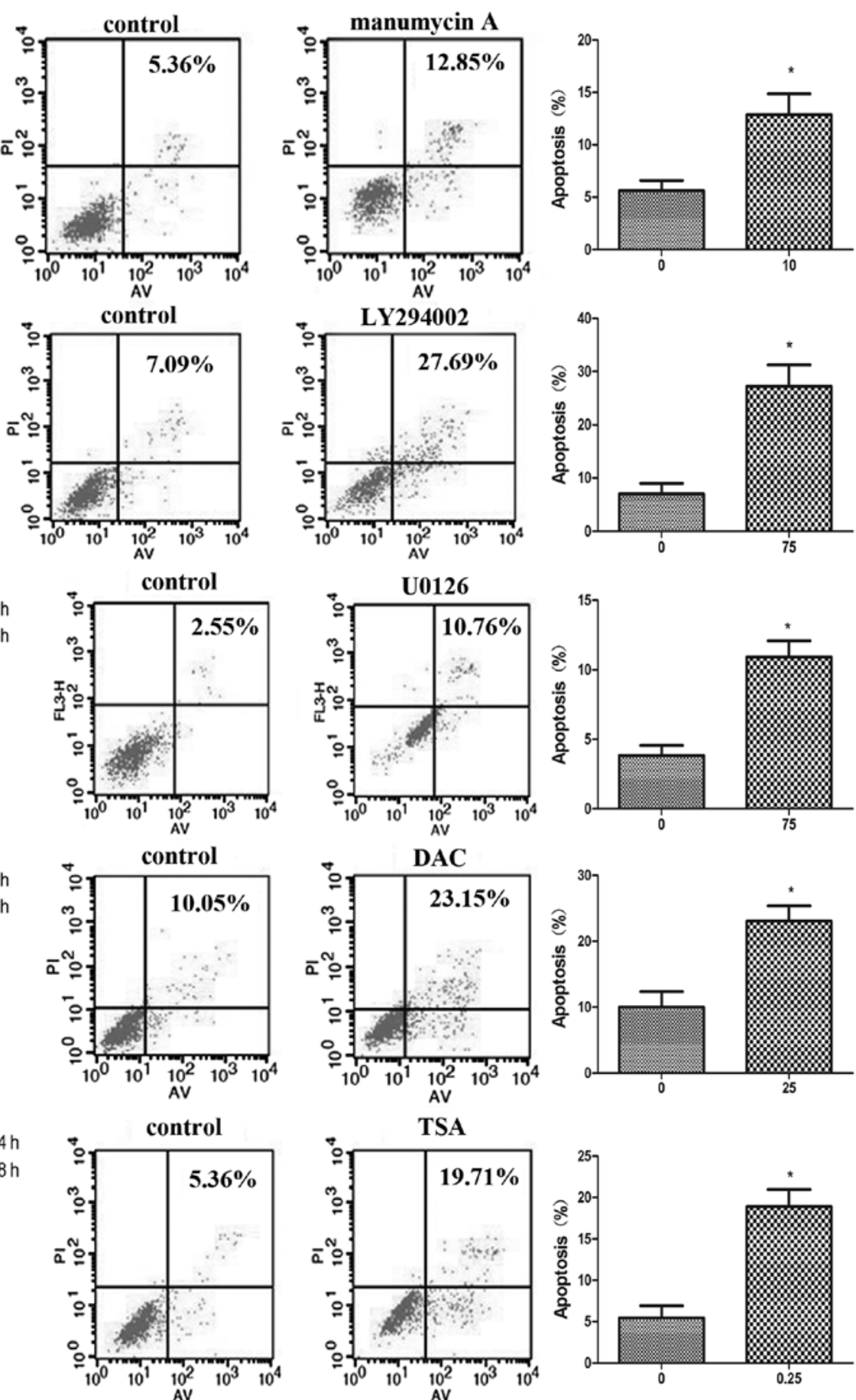

Figure 4. Growth inhibition and apoptosis in HuCCT1 cells. (A) Cell viability: cells were treated with indicated concentrations of manumycin A for 24 and $48 \mathrm{~h}$. Apoptosis: cells were treated with $10 \mu \mathrm{mol} / 1$ manumycin A for $24 \mathrm{~h}$. (B) Cell viability: cells were treated with indicated concentrations of LY294002 for 48 and $96 \mathrm{~h}$. Apoptosis: cells were treated with $75 \mu \mathrm{mol} / 1 \mathrm{LY} 294002$ for $24 \mathrm{~h}$. (C) Cell viability: cells were treated with indicated concentrations of U0126 for 48 and $96 \mathrm{~h}$. Apoptosis: cells were treated with $75 \mu \mathrm{mol} / 1$ U0126 for $24 \mathrm{~h}$. (D) Cell viability: cells were treated with indicated concentrations of DAC for 24 and $48 \mathrm{~h}$. Apoptosis: cells were treated with $25 \mu \mathrm{mol} / 1 \mathrm{DAC}$ for $24 \mathrm{~h}$. (E) Cell viability: cells were treated with indicated concentrations of TSA for 24 and $48 \mathrm{~h}$. Apoptosis: cells were treated with $0.25 \mu \mathrm{mol} / 1 \mathrm{TSA}$ for $24 \mathrm{~h}$. ${ }^{\text {" }} \mathrm{P}<0.05$.

carcinoma (16,32-35). To our knowledge, our study is the first to analyze the effects of LY294002 and U0126 on the proliferation, migration, and apoptosis of HuCCT1 cells. We demonstrated that LY294002 and U0126 inhibited HuCCT1 proliferation in a dose- and in a time-dependent manner, in agreement with previous studies (16,32-35) (Fig. 4B and C). Although extrahepatic cholangiocarcninoma cells and gall- bladder carcinoma cells have been treated with the inhibitors DAC or TSA in previous studies $(36,37)$, their effect on intrahepatic cholangiocarcninoma cells is unknown. In the present study, we made a novel observation that DAC inhibited the growth of HuCCT1 cells in a dose- and time-dependent manner (Fig. 4D). Our results were in agreement with those of a previous study showing that DAC inhibited the growth of 


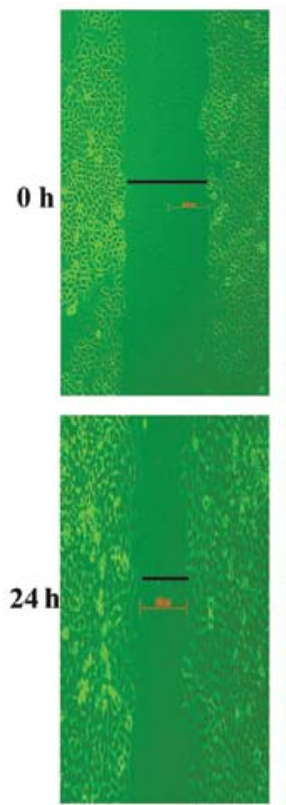

control
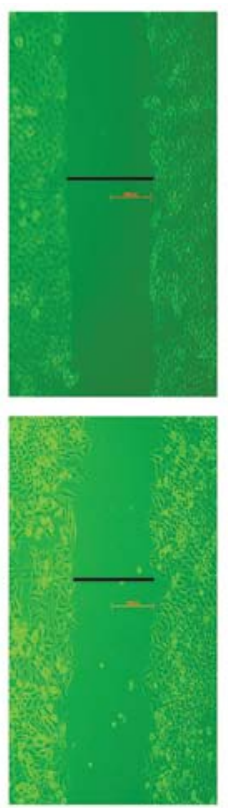

manumycin A
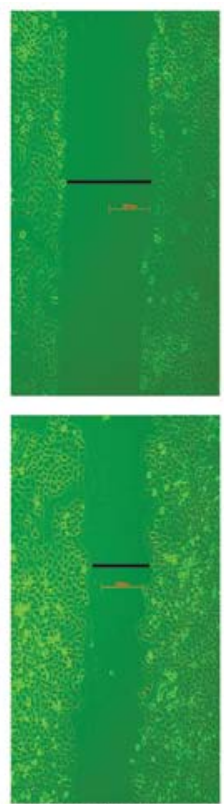

LY294002
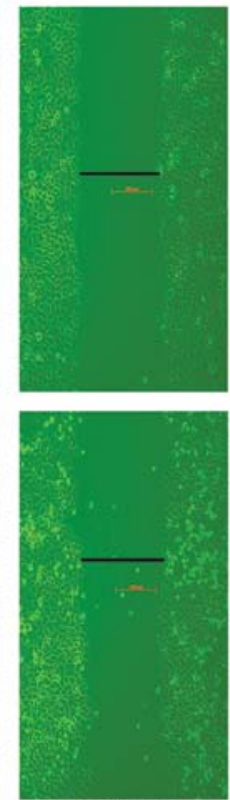

U0126
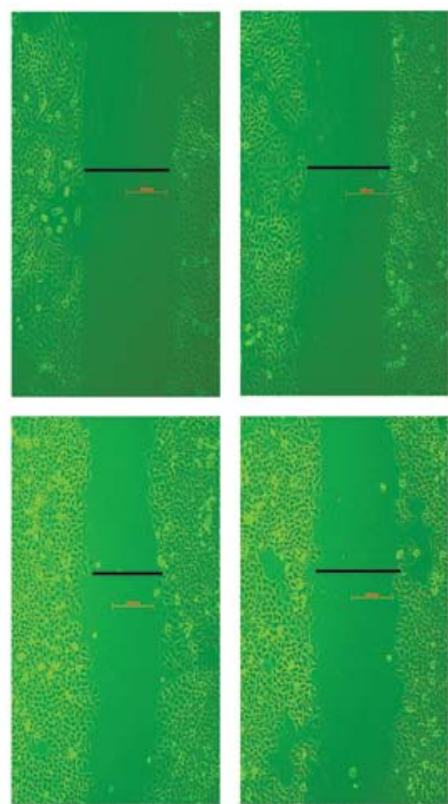

DAC

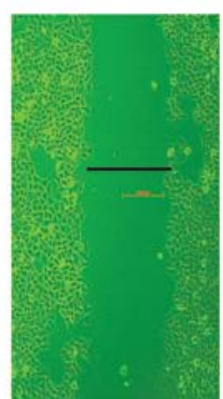

TSA

Figure 5. Migration of HuCCT1 cells measured in wound healing experiments after the treatment with inhibitors, compared with migration of untreated cells.

QBC939 cells in a dose- and time-dependent manner (36). We also found that TSA inhibited the growth of HuCCT1 cells in a dose- and time-dependent manner (Fig. 4E).

Inhibitor-induced increase in apoptosis of cholangiocarcinoma cell lines. Our results showed that manumycin A, LY294002, U0126, DAC and TSA were able to inhibit the growth of HuCCT1 cells. We further demonstrated that inhibitor-treated HuCCT1 cells underwent increased apoptosis compared with the untreated cells. Manumycin A(10 $\mu \mathrm{mol} / \mathrm{l})$, LY294002 $(75 \mu \mathrm{mol} / 1)$ and U0126 $(75 \mu \mathrm{mol} / 1)$, DAC $(25 \mu \mathrm{mol} / \mathrm{l})$ and TSA $(0.25 \mu \mathrm{mol} / \mathrm{l})$ significantly increased apoptosis of HuCCT1 cells (Fig. 4A, B, C, D and E respectively) compared with untreated cells $(\mathrm{P}<0.05)$.

Inhibition of cell migration by manumycin A, LY294002, U0126, DAC and TSA. Migration is one of the most important characteristics of malignant tumors, especially of cholangiocarcinomas. TPM1 plays an important role in cell motility and migration $(10,11)$. We used specific inhibitors that blocked genetic and epigenetic mechanisms to investigate whether the migration of HuCCT1 cells is affected. HuCCT1 cells were wounded and treated with $5 \mu \mathrm{mol} / 1$ manumycin A, $5 \mu \mathrm{mol} / 1$ LY294002, $20 \mu \mathrm{mol} / 1 \mathrm{U} 0126,25 \mu \mathrm{mol} / 1 \mathrm{DAC}$ and $0.1 \mu \mathrm{mol} / 1$ TSA. Images were recorded at the time of wounding $(0 \mathrm{~h})$ and after $24 \mathrm{~h}$. After $24 \mathrm{~h}$, all the five inhibitors inhibited the migration of HuCCT1 cells in culture (Fig. 5).

\section{Discussion}

It has been previously reported that TPM1 functions as a tumor suppressor gene and is downregulated in cancers originating from the epithelial cells, such as breast cancer, colon cancer and urinary bladder carcinoma (6-8). However, the status and significance of TPM1 expression in cholangiocarcinoma has never been investigated. In the present study, we determined TPM1 expression levels in cholangiocarcinoma tissues and cell lines.

RT-PCR and western blot analyses revealed that TPM1 expression was downregulated in HuCCT1 cells and upregulated in QBC939 cells at the mRNA level and protein level. The HuCCT1 cell line was selected to investigate the mechanism of TPM1 downregulation, because TPM1 expression was significantly downregulted compared to HIBEC and HuCCT1 cell line contains $\mathrm{K}$-ras mutations so that it is the appropriate cell model to investigate the role of ras signaling pathway in downregulating TPM1 expression. However, the discrepancy in TPM1 expression observed in the HuCCT1 and QBC939 cell lines requires further studies.

Contradictory to previous reports on cholangiocarcinoma cell lines, we found that there was no significant difference between TPM1 expression in cancer tissues and adjacent non-cancer tissues. Previous immunohistochemical studies have shown that TPM1 expression decreases in tongue squamous cell carcinoma tissue compared with the normal tongue tissue (27). However, in our study, no significant change in TPM1 expression between the cholangiocarcinoma tissues and adjacent non-cancer tissues was observed. This discrepancy in TPM1 expression in tissues and cell lines may be due to our relative small sample size, or due to the fact that immunohistochemistry was not able to identify the expression change in TPM1. Because HIBEC cells are derived from normal intrahepatic biliary cells, it is desirable to use normal intrahepatic biliary tissues in future immunohistochemical studies.

To further understand the mechanism of TPM1 downregulation in HuCCT1 cells, we investigated the role of the Ras signaling pathway and of the epigenetic mechanisms such as DNA methylation, histone deacetylation and miR-21 upregulation in the regulation of TPM1. Previous studies have 
shown that the ras pathway plays an important role in the downregulation of TPM1 in urinary bladder carcinoma and breast cancer $(8,12)$, Given that the activated Ras mutation had been reported in HuCCT1 cells $(15,16)$, we treated these cells with manumycin A, a specific inhibitor of Ras function. Our study is the first to investigate the effect of manumycin on cholangiocarcinoma cells, and show that TPM1 is significantly upregulated in HuCCT1 cells, indicating that Ras plays an important role in the downregulation of TPM1 in cholangiocarcinoma. Furthermore, our study suggested that the activated MEK/ERK pathway contributes to the downregulation of TPM1, which is in agreement with a previous study showing that the MEK/ERK pathway is involved in TPM1 regulation $(8,21)$. Contradictory to the role of the MEK/ERK pathway, some literature reports conclude that the PI3K/AKT pathway has no effect on the downregulation of TPM1 $(8,21)$; however, we inferred that activated PI3K/AKT pathway upregulated TPM1 expression on the basis of our finding that inhibition of PI3K/AKT pathway downregulated TPM1 expression. Spl-binding sites in the TPM1 promoter have been identified, and Sp1 is known to play an important role in the induction of TPM1 (22). Furthermore, other studies have shown that activated PI3K/Akt can recruit $\mathrm{Sp} 1$ to increase the promoter activity in hepatocellular carcinoma cell lines and clear cell renal cell carcinoma cells $(38,39)$. Thus, these results suggest that inhibition of the PI3K/AKT pathway downregulates TPM1 expression in HuCCT1 cells.

DNA methylation is one of the possible mechanisms that contributes to the downregulation of TPM1 (22). Similarly, histone deacetylation is another mechanism involved in the regulation of TPM1 expression in breast cancer and in melanoma $(6,23)$. In the present study, DAC and TSA were used to treat HuCCT1 cells, both of which upregulated TPM1 expression, indicating that DNA methylation as well as histone deacetylation play an important role in TPM1 expression in cholangiocarcinoma cells. Further studies assessing the methylation of individual CpG sites in the TPM1 promoter before and after DAC treatment are required to shed further light on the regulatory mechanism.

Along with the involvement of DNA methylation and histone deacetylation in the downregulation of TPM1, we also identified TPM1 as a target of miR-21. Many studies have shown that TPM1 is a direct target of miR-21 (25-29), and that miR-21 is upregulated in cholangiocarcinoma (40). In our study, miR-21 was shown to be upregulated in HuCCT1 cells compared with HIBEC cells, indicating that miR-21 may regulate TPM1 expression in HuCCT1 cells. Using miR-21 knockdown to investigate the change in TPM1 expression at the mRNA and protein levels, we demonstrated that miR-21 regulates TPM1 at the protein level, but not at the mRNA level. A luciferase activity assay should be performed in the future to determine whether TPM1 is a direct target of miR-21.

Cholangiocarcinoma is a malignancy of the biliary tract that is highly chemoresistant to chemical agents used in the clinic; however, the mechanisms regulating cholangiocarcinoma growth and resistance to chemotherapy remain poorly understood (40). Our study investigated the inhibitory effect of these chemical agents on HuCCT1 cells. Involvement of the activated Ras mutation in cholangiocarcinoma has been demonstrated $(15,16)$. We used manumycin A for the first time to treat cholangiocarcinoma cells and showed that it could inhibit proliferation, induce apoptosis and inhibit migration of HuCCT1 cells, thus suggesting that manumycin by blocking the function of Ras could serve as an important agent to treat cholangiocarcinoma. Although the use of PI3K/AKT and MEK/ERK inhibitors as anticancer agents has been widely acknowledged, few have made it to clinical trials. Our study indicated that the PI3K/AKT inhibitor LY294002 and the MEK/ERK inhibitor U0126 inhibited the growth and migration of HuCCT1 cells and induced apoptosis, suggesting that these signaling pathway inhibitors may be an optimized therapeutic strategy against cholangiocarcinoma (30). Although inhibitors of DNA methylation and histone deacetylation have been used to treat metastatic gallbladder cancer cells (41) and extrahepatic cholangiocarcinoma cells (37), their effect on intrahepatic cholangiocarcinoma cells was previously unknown, and recent data have shown that DNA methylation and histone deacetylation are promising targets of cancer treatment $(36,37)$. Our results showed that DAC and TSA shortened the survival time of HuCCT1 cells, induced apoptosis, and inhibited cell migration, indicating that DAC and TSA would be promising agents in the treatment of cholangiocarcinoma.

In the present study, investigations to elucidate the underlying mechanisms of TPM1 downregulation were focused on the Ras signaling pathway, showing the involvement of both the Ras downstream signaling pathways, RAS/PI3K/AKT and RAS/MEK/ERK, in TPM1 downregulation. This is the first report suggesting the possible mechanism underlying TPM1 suppression in cholangiocarcinoma; in particular, this is the first attempt to block the function of Ras in order to investigate the role of the Ras signal pathway in cholangiocarcinoma. In addition to genetic mutation mechanisms, our study also found that the three important epigenetic mechanisms, namely DNA methylation, histone deacetylation, and miR-21 upregulation were all involved in the downregulation of TPM1 in cholangiocarcinoma. In conclusion, the mechanism of TPM1 downregulation is very complicated, and inhibitors targeting genetic signal pathways or epigenetic mechanisms may be promising agents to treat cholangiocarcinoma in the future.

\section{Acknowledgements}

This study was supported by the Department of Health of the JiangSu Province Fund.

\section{References}

1. Khan SA, Thomas HC, Davidson BR and Taylor-Robinson SD: Cholangiocarcinoma. Lancet 366: 1303-1304, 2005.

2. Hong K and Geschwind JF: Locoregional: intra-arterial therapies for unresectable intrahepatic cholangiocarcinoma. Semin Oncol 37: 110-117, 2010.

3. Ramage JK, Donaghy A, Farrant JM, Iorns R and Williams R: Serum tumor markers for the diagnosis of cholangiocarcinoma in primary sclerosing cholangitis. Gastroenterology 108: 865-869, 1995.

4. Schevzov G, Whittaker SP, Fath T, Lin JJ and Gunning PW: Tropomyosin isoforms and reagents. Bioarchitecture 1: 135-164, 2011.

5. Lin JJ, Warren KS, WamboldtDD, Wang T and Lin JL: Tropomyosin isoforms in non-muscle cells. Int Rev Cytol 170: 1-38, 1997.

6. Bharadwaj S and Prasad L: Tropomyosin-1, a novel suppressor of cellular transformation is downregulated by promoter methylation in cancer cells. Cancer Lett 183: 205-213, 2002. 
7. Raval GN, Bharadwaj S, Levine EA, Willingham MC, Geary RL, Kute T and Prasad GL: Loss of expression of tropomyosin-1, a novel class II tumor suppressor that induces anoikis, in primary breast tumors. Oncogene 22: 6194-6203, 2003.

8. Pawlak G, McGarvey TW, Nguyen TB, Tomaszewski JE, Puthiyaveettil R, Malkowicz SB and Helfman DM: Alterations in tropomyosin isoform expression in human transitional cell carcinoma of the urinary bladder. Int J Cancer 110: 368-373, 2004.

9. Yager ML, Hughes JA, Lovicu FJ, Gunning PW, Weinberger RP and O'Neill GM: Functional analysis of the actin binding protein, tropomyosin 1, in neuroblastoma. Br J Cancer 89: 860-863, 2003

10. Choi C, Kim D, Kim S, Jeong S, Song E and Helfman DM: From skeletal muscle to cancer: insights learned elucidating the function of tropomyosin. J Struct Biol 177: 63-69, 2012.

11. Mahadev K, Raval G, Bharadwaj S, et al: Suppression of the transformed phenotype of breast cancer by tropomyosin-1. Exp Cell Res 279: 40-51, 2002.

12. Bakin AV, Safina A, Rinehart C, Daroqui C, Darbary H and Helfman DM: A critical role of tropomyosins in TGF-beta regulation of the actin cytoskeleton and cell motility in epithelial cells. Mol Biol Cell 15: 4682-4694, 2004.

13. Xu RF, Sun JP, Zhang SR, et al: KRAS and PIK3CA but not BRAF genes are frequently mutated in Chinese cholangiocarcinoma patients. Biomed Pharmacother 65: 22-26, 2011.

14. O'Dell MR, Huang JL, Whitney-Miller CL, et al: Kras(G12D) and p53 mutation cause primary intrahepatic cholangiocarcinoma. Cancer Res 15: 1557-1567, 2012.

15. Imai $M$ and Takahashi N: Growth inhibition and mechanism of action of p-dodecylaminophenol against refractory human pancreatic cancer and cholangiocarcinoma. Bioorg Med Chem 20: 2520-2526, 2012

16. Jinawath A, Akiyama Y, Sripa B and Yuasa Y: Dual blockade of the Hedgehog and ERK1/2 pathways coordinately decreases proliferation and survival of cholangiocarcinoma cells. J Cancer Res Clin Oncol 133: 271-278, 2007.

17. Hara M, Akasaka K, Akinaga S, et al: Identification of Ras farnesyltransferase inhibitors by microbial screening. Proc Natl Acad Sci USA 90: 2281-2285, 1993.

18. Nagase T, Kawata S, Tamura S, et al: Inhibition of cell growth of human hepatoma cell line (Hep G2) by a farnesyl protein transferase inhibitor: a preferential suppression of ras farnesylation. Int J Cancer 65: 620-626, 1996.

19. Kainuma O, Asano T, Hasegawa M, Kenmochi T, Nakagohri T, Tokoro $\mathrm{Y}$ and Isono K: Inhibition of growth and invasive activity of human pancreatic cancer cells by a farnesyltransferase inhibitor, manumycin. Pancreas 15: 379-383, 1997.

20. Ito T, Kawata S, Tamura S, et al: Suppression of human pancreatic cancer growth in BALB/c nude mice by manumycin, a farnesyl:protein transferase inhibitor. Jpn J Cancer Res 87: 113-116, 1996

21. Shields JM, Mehta H, Pruitt K and Der CJ: Opposing roles of the extracellular signal-regulated kinase and p38 mitogen-activated protein kinase cascades in Ras-mediated downregulation of tropomyosin. Mol Cell Biol 22: 2304-2317, 2002.

22. Varga AE, Stourman NV, Zheng Q, et al: Silencing of the Tropomyosin-1 gene by DNA methylation alters tumor suppressor function of TGF-beta. Oncogene 24: 5043-5052, 2005.

23. Liu S, Ren S, Howell P, Fodstad O and Riker AI: Identification of novel epigenetically modified genes in human melanoma via promoter methylation gene profiling. Pigment Cell Melanoma Res 21: 545-558, 2008

24. Kawahigashi Y, Mishima T, Mizuguchi Y, et al: MicroRNA profiling of human intrahepatic cholangiocarcinoma cell lines reveals biliary epithelial cell-specific microRNAs. J Nihon Med Sch 76: 188-197, 2009.
25. Zhu S, Si ML, Wu H and Mo YY: MicroRNA-21 targets the tumor suppressor gene tropomyosin 1 (TPM1). J Biol Chem 282: 14328-14336, 2007

26. Zhu S, Wu H, Wu F, Nie D, Sheng S and Mo YY: MicroRNA-21 targets tumor suppressor genes in invasion and metastasis. Cell Res 18: 350-359, 2008

27. Li J, Huang H, Sun L, et al: MiR-21 indicates poor prognosis in tongue squamous cell carcinomas as an apoptosis inhibitor. Clin Cancer Res 15: 3998-4008, 2009.

28. Li T, Li D, Sha J, Sun P and Huang Y: MicroRNA-21 directly targets MARCKS and promotes apoptosis resistance and invasion in prostate cancer cells. Biochem Biophys Res Commun 383: 280-285, 2009

29. Dong CG, Wu WK, Feng SY, Wang XJ, Shao JF and Qiao J: Co-inhibition of microRNA-10b and microRNA-21 exerts synergistic inhibition on the proliferation and invasion of human glioma cells. Int J Oncol 41: 1005-1012, 2012.

30. Schmitz KJ, Lang H, Wohlschlaeger J, Sotiropoulos GC, Reis H, Schmid KW and Baba HA: AKT and ERK1/2 signaling in intrahepatic cholangiocarcinoma. World J Gastroenterol 13: 6470-6477, 2007.

31. Leelawat K, Leelawat S, Narong S and Hongeng S: Roles of the MEK1/2 and AKT pathways in CXCL12/CXCR4 induced cholangiocarcinoma cell invasion. World J Gastroenterol 13: 1561-1568, 2007.

32. Leelawat K, Narong S, Udomchaiprasertkul W, Leelawat S and Tungpradubkul S: Inhibition of PI3K increases oxaliplatin sensitivity in cholangiocarcinoma cells. Cancer Cell Int 9: 3, 2009.

33. Menakongka A and Suthiphongchai T: Involvement of PI3K and ERK1/2 pathways in hepatocyte growth factor-induced cholangiocarcinoma cell invasion. World J Gastroenterol 16: 713-722, 2010.

34. Wu T, Leng J, Han C and Demetris AJ: The cyclooxygenase-2 inhibitor celecoxib blocks phosphorylation of Akt and induces apoptosis in human cholangiocarcinoma cells. Mol Cancer Ther 3: 299-307, 2004.

35. Matsumoto K, Nagahara T, Okano J and Murawaki Y: The growth inhibition of hepatocellular and cholangiocellular carcinoma cells by gemcitabine and the roles of extracellular signal-regulated and checkpoint kinases. Oncol Rep 20: 863-872, 2008.

36. Liu XF, Jiang H, Zhang CS, Yu SP, Wang ZQ and Su HL: Targeted drug regulation on methylation of p53-BAX mitochondrial apoptosis pathway affects the growth of cholangiocarcinoma cells. J Int Med Res 40: 67-75, 2012.

37. Xu LN, Wang X and Zou SQ: Effect of histone deacetylase inhibitor on proliferation of biliary tract cancer cell lines. World J Gastroenterol 14: 2578-2581, 2008.

38. Yin $\mathrm{P}, \mathrm{Zhao} \mathrm{C}, \mathrm{Li} \mathrm{Z}$, et al: $\mathrm{Sp} 1$ is involved in regulation of cystathionine gamma-lyase gene expression and biological function by PI3K/Akt pathway in human hepatocellular carcinoma cell lines. Cell Signal 24: 1229-1240, 2012.

39. Tang SW, Yang TC, Lin WC, Chang WH, Wang CC, Lai MK and Lin JY: Nicotinamide N-methyltransferase induces cellular invasion through activating matrix metalloproteinase-2 expression in clear cell renal cell carcinoma cells. Carcinogenesis 32 : 138-145, 2011.

40. Meng F, Henson R, Lang M, et al: Involvement of human micro-RNA in growth and response to chemotherapy in human cholangiocarcinoma cell line. Gastroenterology 130: 2113-2129, 2006.

41. Wehbe H, Henson R, Meng F, Mize-Berge J and Patel T: Interleukin- 6 contributes to growth in cholangiocarcinoma cells by aberrant promoter methylation and gene expression. Cancer Res 66: 10517-10524, 2006. 